\title{
On the use of multilayer Laue lenses with X-ray free electron lasers
}

\section{Mauro Prasciolu, Kevin Murray, Nikolay Ivanov, Holger Fleckenstein, Martin Domaracký, et al.}

Mauro Prașciolu, Kevin T. Murray, Nikolay Ivanov, Holger Fleckenstein, Martin Domaracký, Luca Gelisio, Fabian Trost, Kartik Ayyer, Dietrich Krebs, Steve Aplin, Salah Awel, Ulrike Boesenberg, Grega Belšak, Anton Barty, Armando D. Estillore, Matthias Fuchs, Yaroslav Gevorkov, Joerg Hallmann, Chan Kim, Juraj Knoška, Jochen Küpper, Chufeng Li, Wei Lu, Valerio Mariani, Andrew J. Morgan, Johannes Möller, Anders Madsen, Dominik Oberthür, Gisel E. Peña Murillo, David A. Reis, Markus Scholz, Božidar Šarler, Pablo VillanuevaPerez, Oleksandr Yefanov, Kara A. Zielinski, Alexey Zozulya, Henry N. Chapman, Saša Bajt, "On the use of multilayer Laue lenses with X-ray free electron lasers," Proc. SPIE 11886, International Conference on X-Ray Lasers 2020, 118860M (8 July 2021); doi: 10.1117/12.2592229 


\title{
On the Use of Multilayer Laue Lenses with X-ray Free Electron Lasers
}

\author{
Mauro Prasciolua, Kevin T. Murray ${ }^{\mathrm{a}}$, Nikolay Ivanov ${ }^{\mathrm{a}}$, Holger Fleckenstein ${ }^{\mathrm{b}}$, Martin Domarackýb, \\ Luca Gelisio $^{\mathrm{b}}$, Fabian Trost ${ }^{\mathrm{b}}$, Kartik Ayyer ${ }^{\mathrm{c}}$, Dietrich Krebs ${ }^{\mathrm{b}, \mathrm{d}}$, Steve Aplin ${ }^{\mathrm{e}}$, Salah Awel ${ }^{\mathrm{b}}$, \\ Ulrike Boesenberg ${ }^{\mathrm{e}}$, Grega Belšak ${ }^{\mathrm{f}}$, Anton Barty ${ }^{\mathrm{b}}$, Armando D. Estillore ${ }^{\mathrm{b}}$, Matthias Fuchs ${ }^{\mathrm{g}}$, \\ Yaroslav Gevorkov $^{\text {b,h }}$, Joerg Hallmann ${ }^{\mathrm{e}}$, Chan Kim ${ }^{\mathrm{e}}$, Juraj Knoška ${ }^{\mathrm{b}, \mathrm{d}}$, Jochen Küpper ${ }^{\mathrm{b}, \mathrm{d}, \mathrm{i}}$, \\ Chufeng Li ${ }^{\mathrm{b}}$, Wei Lu ${ }^{\mathrm{e}}$, Valerio Mariani ${ }^{\mathrm{b}}$, Andrew J. Morgan ${ }^{\mathrm{j}}$, Johannes Möller ${ }^{\mathrm{e}}$, Anders Madsen ${ }^{\mathrm{e}}$, \\ Dominik Oberthür ${ }^{\mathrm{b}}$, Gisel E. Peña Murillo ${ }^{\mathrm{b}, \mathrm{d}}$, David A. Reis ${ }^{\mathrm{k}, \mathrm{l}}$, Markus Scholz ${ }^{\mathrm{e}}$, Božidar Šarler ${ }^{\mathrm{f}, \mathrm{m}}$, \\ Pablo Villanueva-Perez ${ }^{\mathrm{n}}$, Oleksandr Yefanov ${ }^{\mathrm{b}}$, Kara A. Zielinski ${ }^{\mathrm{b}}$, Alexey Zozulya ${ }^{\mathrm{e}}$, \\ Henry N. Chapman ${ }^{\text {b,d,i }}$, and Saša Bajt*a,i \\ a Deutsches Elektronen-Synchrotron DESY, Notkestraße 85, 22607 Hamburg, Germany; ' CFEL, \\ Center for Free-Electron Laser Science, Deutsches Elektronen-Synchrotron DESY, Notkestraße 85, \\ 22607 Hamburg, Germany, 'Max Planck Institute for the Structure and Dynamics of Matter, \\ Hamburg 22761, Germany, d Department of Physics, Universität Hamburg, Luruper Chaussee 149, \\ 22761 Hamburg, Germany, ${ }^{e}$ European X-ray Free-Electron Laser Facility, Holzkoppel 4, 22869

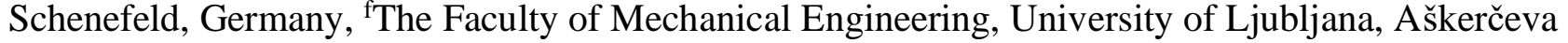 \\ 6, 1000 Ljubljana, Slovenia, 'Department of Physics and Astronomy, University of Nebraska- \\ Lincoln, Lincoln, Nebraska 68588, USA, hVision Systems, Hamburg University of Technology,

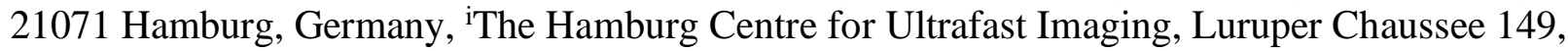 \\ 22761 Hamburg, Germany, ${ }^{j}$ School of Physics, University of Melbourne, Parkville, Victoria 3010, \\ Australia, ${ }^{\mathrm{k} S t a n f o r d ~ P U L S E ~ I n s t i t u t e, ~ S L A C ~ N a t i o n a l ~ A c c e l e r a t o r ~ L a b o r a t o r y, ~ M e n l o ~ P a r k, ~}$ \\ California 94025, USA, 'Stanford Institute for Materials and Energy Sciences, SLAC National \\ Accelerator Laboratory, Menlo Park, California 94025, USA, mInstitute of Metals and Technology, \\ Lepi pot 11, 1000 Ljubljana, Slovenia, " Synchrotron Radiation Research and NanoLund, Lund \\ University, Sölvegatan 14A, Lund, Sweden.
}

\begin{abstract}
We report on the use of multilayer Laue lenses to focus the intense X-ray Free Electron Laser (XFEL) beam at the European XFEL to a spot size of a few tens of nanometers. We present the procedure to align and characterize these lenses and discuss challenges working with the pulse trains from this unique $\mathrm{x}$-ray source.
\end{abstract}

Keywords: x-rays, multilayer Laue lenses, XFEL, optics

\section{INTRODUCTION}

X-ray free electron lasers distinguish themselves from other x-ray sources with their extremely short pulses, high transverse coherence and high peak powers. These properties opened up new research areas including single shot imaging of biological samples, imaging the dynamics of matter, creating matter under extreme conditions and studying nonlinear optical processes in the hard x-ray regime. Nonlinear Compton scattering [1], for example, occurs at extreme intensities when two incoming x-ray photons of photon energy $E$ simultaneously interact with an atom to convert into a single x-ray photon that is energetically red-shifted in comparison to $2 E$. The generation of a photon at precisely $2 E$

*sasa.bajt@desy.de; phone +49 408998 2082;

International Conference on X-Ray Lasers 2020, edited by Davide Bleiner,

Proc. of SPIE Vol. 11886, 118860M · (C) 2021 SPIE · CCC code:

0277-786X/21/\$21 - doi: $10.1117 / 12.2592229$ 
corresponds to the process of second harmonic generation, which is well known in nonlinear optics and has also been observed in the x-ray regime [2]. X-ray and optical photons can also combine at high intensities and in the presence of matter in difference- and sum-frequency generation [3] and other wave-mixing processes such as parametric down

conversion [4]. All these experiments also require very high fluence, which can be increased by focusing the XFEL beams to a smaller spot. We wish to investigate the generation of high-intensity beams at XFEL sources using multilayer Laue lenses (MLLs) [5]. This new generation of highly efficient and robust x-ray optical elements has been designed to operate under the full beam conditions of these sources [6,7]. Part of this work was done during the commissioning of the MID $[8,9]$ beamline of the European XFEL (Schenefeld, Germany) [10,11] in two experimental runs (2200 and 2543).

In general, focusing x-ray beams to a very small spot, below $10 \mathrm{~nm}$, is challenging. It requires optics with little or no wavefront aberrations. Optical elements used with XFEL beams have to be also extremely robust to survive and perform reliably when exposed to high power beams. XFEL beams are typically focused with compound refractive lenses (CRLs) or reflective mirrors. To increase the numerical aperture (NA) of CRLs, which is needed to achieve very small spot size, many lenses need to be stacked behind each other [12]. This however, also reduces their efficiency. Furthermore, CRLs have strong chromatic aberrations so their ability to focus to extremely small spot sizes is limited [13]. Reflective mirrors, such as Kirkpatrick-Baez (KB) mirrors, which operate at grazing incidence, can accept the full beam. Due to their operation at grazing incidence, the interaction with the X-ray beam is spread over a large area. This minimizes the power per area or dose to the material and protects the mirror from radiation damage. The reflectivity and efficiency of KB mirror system can be very high. However, substrates and coatings are required that have nearly perfect surface figure and extremely low surface roughness [14]. Diffraction-based optics, such as Fresnel zone plates, can also be used to focus XFEL beams [1517]. They also have chromatic aberrations, but their effects are generally smaller than for CRLs due to their shorter focal lengths and lower dispersion. Since these lenses consist of nanostructures printed on x-ray transparent membranes, they are fragile. Theoretical simulations predicted that rapid temperature fluctuations of many hundred degrees Kelvin lead in stress and/or strain changes followed by a failure when exposed to the direct beam [18]. However, the response of zone plates is also strongly dependent on the materials used in these nanostructures. For example, typical zone plate materials, such as gold and tungsten, cannot survive long in an intense XFEL beam. But combining high-Z metal (Ir) with excellent heat dissipation of low-Z material (diamond), as demonstrated with Ir-filled diamond zone plates, leads to increased radiation hardness [15]. Such zone plates could withstand unfocused XFEL beam at LCLS (SLAC, USA) but under prefocused XFEL beam their damage threshold was surpassed. It was reported that the LCLS beam at 4 and $8 \mathrm{keV}$ photon energies and a beam size of $2 \times 2 \mathrm{~mm}^{2}$ [19] were not detrimental for grating nanostructures made of pure diamond. This beam size was much larger than the typical areas of zone plates. Making zone plates this large is possible, but requires an increase in the number of zones which in turn increases its chromatic aberration, thus reducing the tolerable bandwidth. In this paper we report about focusing XFEL beams with MLLs. These diffractive optics have much higher diffraction efficiency as compared to Fresnel zone plates and combined with high NA could potentially focus XFEL beam to intensities required to study nonlinear processes such as mentioned above.

\section{MULTILAYER LAUE LENSES}

All MLLs used in this study were prepared in our laboratory with a custom-built magnetron sputtering system. The multilayers consisted of silicon carbide (SiC) and tungsten carbide (WC), which form sharp, smooth and stable interfaces $[6,7,20]$. The multilayer, which was $>105 \mu \mathrm{m}$ thick, consisted of 10048 bilayers and was deposited on a polished Si wafer substrate with initial roughness of about $0.15 \mathrm{~nm}$ rms. The substrate was moved between the two sputter targets in the process of spinning around its own axis while the layer thickness was controlled by varying the platter velocity.

Similar to Fresnel zone plates, the resolution of an MLL lens is determined by the finest layer. Tungsten carbide/silicon carbide (WC/SiC) multilayer systems have remarkably low interfacial roughness and can form nanometer or even subnanometer periods as previously demonstrated for periodic multilayers [21,22]. However, the variation in period that produces the focusing behavior in MLLs leads to stress and interface roughness variation in the deposited nanostructure. Also, the total multilayer structure is hundreds of times thicker than the multilayers usually used for reflective coatings. We overcame these challenges using new materials and by optimizing the sputtering process as reported before [7]. The refractive carbide materials used here produce multilayer structures that are extremely thermally stable and retain their period when heated up to temperatures of $800^{\circ} \mathrm{C}$ [6]. A high aspect ratio of the period of the finest layer to the thickness of the lens (in the direction of beam transmission) is needed to achieve high diffraction efficiency. An aspect ratio of several thousand is needed in x-ray regime above about $10 \mathrm{keV}$. Such high aspect ratio structures can easily be achieved 
when slicing an MLL from the deposited structure. Diffraction efficiencies above $80 \%$ were achieved for energies of 17 $20 \mathrm{keV}$ [23] and even higher efficiencies can be reached at higher energies. To obtain high efficiency over the entire lens, it is critical that each layer is also correctly tilted to satisfy Bragg's law in each position along the lens. We developed a simple method to achieve this wedging of the layers by affixing a straight-edged mask at a pre-selected height above the substrate and spinning it together with the substrate during the deposition process [24]. A desired thickness profile is then formed in the penumbra of the mask. Because it takes a very long time to deposit such thick multilayers ( $~ 6$ days to prepare $105 \mu \mathrm{m}$ thick multilayer) we maximized the deposition output by utilizing two masks placed at different heights. Consequently, we could prepare lenses optimized for the same energy but of two different focal lengths during the same deposition run. After the deposition was finished the multilayer thickness profiles were determined by making "depth soundings" of the structure at a number of positions using a focused ion beam (FIB) [24]. This was used to identify the positions where layer spacing and layer tilt curvature matched the design. MLLs were cut using a FIB. This took several days per MLL due to their large thickness. Final extraction and transfer of each MLL was performed with a nanomanipulator (Omniprobe ${ }^{\circledR}$ ). Each MLL was attached to the corner of a $100 \mu \mathrm{m}$ thick diamond wafer, welded on the side with two Pt dots (by electron-induced deposition) and finally thinned using the ion beam to an optical thickness that would give the optimal diffraction efficiency.

Experiments reported here were performed at the MID instrument [25] of European XFEL during experimental runs 2200 (April 2019) and 2543 (October 2019) at $8.9 \mathrm{keV}$ and $10.1 \mathrm{keV}$ photon energies, respectively. MLLs optimized for these two energies were designed to focus x-rays in ideal case (unaberrated lenses) to $15 \mathrm{~nm}$ with focal lengths of 6 and $7 \mathrm{~mm}$. Since an MLL deposited on a flat substrate focuses x-rays only in one direction, two MLLs positioned orthogonal to each other, are needed to achieve a 2D focusing. Precise alignment, including astigmatism correction [26], was achieved by mounting each lens on a separate hexapod with six degrees of freedom. Focusing was achieved with a pair of MLLs optimized for the same energy with two different focal lengths. The multilayer, from which these MLLs were prepared, was deposited starting with the thinnest and ending with the thickest layers. This took advantage of the smooth substrate. Such a structure diffracts x-rays away from the plane of the substrate. This off-axis design simplifies separating and blocking the direct beam and the two diffracted beams coming from horizontal and vertical lenses.

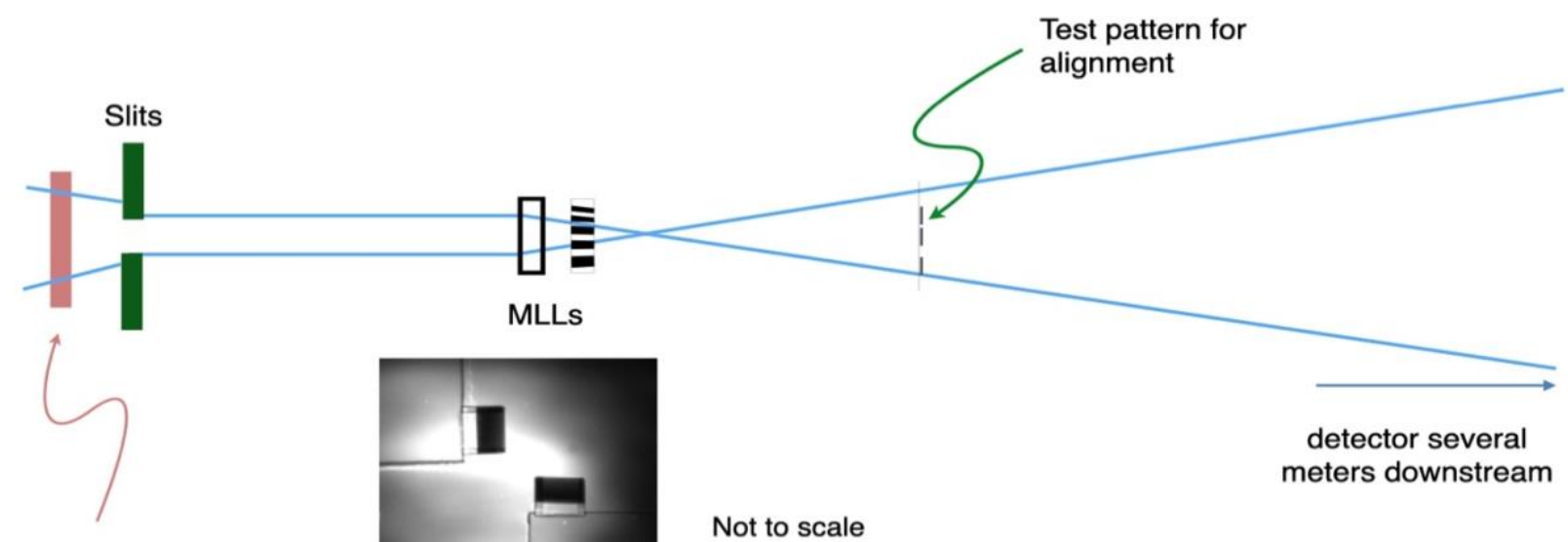

Attenuators

Figure 1. Schematic of the experimental setup showing the major components. The image below the MLLs shows horizonal and vertical focusing MLLs under Bragg condition (black) when illuminated by XFEL beam as seen with x-ray eye detector placed downstream the lenses.

\section{Experimental setup}

The approximate dimensions of the MLLs were $100 \mu \mathrm{m}$ (height) x $125 \mu \mathrm{m}$ (width) x $4.5 \mu \mathrm{m}$ (optical thickness). Such large MLLs are needed to capture the largest possible part of highly coherent XFEL beam, without having to pre-focus it to 
damaging intensities. In the 2200 experimental run we worked with $8.9 \mathrm{keV} x$-rays and the beam was collimated and slightly pre-focused $\left(\sim 300 \times 300 \mu \mathrm{m}^{2}\right)$ using only one set of CRLs. Additional beam size reduction and removal of stray scattering and tails of the beam was accomplished with a set of slits. Nevertheless, the slitted beam over-filled the MLLs thus causing the beam to illuminate not only the lenses but also their supporting structures. Due to beam pointing instabilities and problems with data acquisition, measurements and data analysis were very difficult. In our second campaign (experimental run 2543) we worked with $10.1 \mathrm{keV}$ x-rays. The XFEL beam was collimated and pre-focused with two sets of CRLs. The beam illuminating MLLs was at least three times more intense as compared to experimental run 2200 and an additional pinhole was placed upstream from MLLs (Fig. 1). The x-ray beam path at the MID beamline was in vacuum except in the region surrounding our equipment, of about $1.2 \mathrm{~m}$ length. This region included an upstream pinhole, the set of two MLLs, a sample, removable x-ray eye detector and optical microscope, and a beam stop.

During the initial alignment procedure, the intensity of the XFEL beam was attenuated by several orders of magnitude. An $\mathrm{x}$-ray eye detector was placed along the beam path behind the MLLs. An MLL lens in the beam path will transmit most of the incident $\mathrm{x}$-rays since it is only about $4.5 \mu \mathrm{m}$ thick. However, once it is tilted to the Bragg angle it becomes completely black because it efficiently diffracts most of the x-rays away from the optical axis. This is the case for both lenses in Fig. 1. In this image the lenses are still far apart from each other. During alignment, they are overlapped as visualized with the $\mathrm{x}$-ray eye detector, to ensure that the x-rays diffracting from the first lens fall on the second, orthogonally placed lens. As an alignment sample we used a structure made out of gold using optical lithography. It consisted of a $400 \mathrm{~nm}$ thick gold dot ( $8 \mu \mathrm{m}$ diameter) surrounded by five $4 \mu \mathrm{m}$ wide concentric rings, with increasing diameter. This structure was printed directly on a $200 \mu \mathrm{m}$ diamond wafer and placed close to the focal plane. It was placed downstream of the MLL focus so that a highly magnified image of this structure was projected on the AGIPD (Adaptive Gain Integrating Pixel Detector) [27] detector. AGIPD is high dynamic range pixel array detector with a $4.5 \mathrm{MHz}$ frame rate. The projection image was used to fine tune the alignment, correct the astigmatism (observable as a different magnification of the image in the horizontal and vertical directions) and to determine the focal plane. The AGIPD detector was $4 \mathrm{~m}$ away from the interaction (focus) region. It was under vacuum and the flight path was extended in front of the detector, starting at about $80 \mathrm{~cm}$ from the focal plane with an entrance aperture by an off-axis $40 \mathrm{~mm}$ diameter, $\sim 770 \mu \mathrm{m}$ thick, water cooled, diamond window. In addition to the beam stop that blocked the direct and the single first order diffracted beams, a $300 \mu \mathrm{m}$ thick Si was mounted directly on the diamond window to attenuate focused beam being accepted by AGIPD detector.

\section{RESULTS AND DISCUSSION}

\section{Optics characterization and results}

The wavefronts of the MLLs used in this experiment were pre-characterized using ptychographic x-ray speckle tracking (PXST). More details about the theory [28], experimental application [29] and a software code [30] for PXST can be found elsewhere. The method relies upon the ability to nicely visualize aberrations of the lens by making a projection image of a small sample placed just slightly outside the focus. This is a simple way to form magnified high-resolution images. In order to accurately map out any wavefront distortions of the beam illuminating the sample, the sample should have many small features. The sample is scanned around while magnified holographic images are collected on the detector. The image of the whole sample can then be formed by stitching together many such illuminated patches. However, if the lenses are not perfect (the wavefront not being a perfect sphere) distortions in the image are revealed when images do not perfectly overlap when these small patches are stitched together. So, when we construct the stitched image, we have to "undistort" each projected image so that they match up in the overlapping regions. This is just ptychography, but now applied in a geometric sense, rather than through diffraction. This map of the distortions represents the phase gradient, which after integration gives us the wavefront map of the lens. The focus size and distribution can be extracted once we perform a Fourier transform of the lens wavefront map and backpropagate the wavefront to the focal plane. We found that the phase of two orthogonally placed MLLs is separable [7] and the phase of each lens is similar to the other, which is to be expected since the lenses were prepared the same way. Based on our PXST characterization $[28,29,30]$ the achieved focus in the focal plane was $26 \mathrm{~nm}$. Our estimation of the intensity in the focus is $3.4 \times 10^{18} \mathrm{~W} / \mathrm{cm}^{2}$.

\section{Lens performance under pulse trains}

Here we show the performance of one set of lenses that we were testing by increasing the intensity of the incoming $\mathrm{x}$-ray beam and the number of pulses per train at a pulse energy of $\sim 1.1 \mathrm{~mJ}$. The frequency of pulse trains was $10 \mathrm{~Hz}$, and the 
pulses within a train were separated by either $0.9 \mu$ s or $3.5 \mu$ s. After the MLLs were aligned they were used with single pulse trains and $92 \%$ beamline transmission for 6 hours. Apart from beam-pointing instability no problems were observed. In one study we performed a series of runs in which the number of pulses per train were increased from 1 to 2 , $3,4,5,6,7,10,20$ and 30 pulses per train, all with a pulse separation of $3.5 \mu \mathrm{s}$. The increase in the number of pulses per train was accompanied with an increase of $\mathrm{x}$-ray intensity (transmission) from $9 \%$ to $92 \%$ at 5 pulses per train to a final working condition of 30 pulses per train and $23.5 \%$ transmission. At this condition we saw that the diffraction efficiency of the MLLs changed over the course of a pulse train, and that this variation was reproducible from train to train. Figures 2 and 3 show snapshots of how the magnified pupil of the MLLs, as seen on AGIPD detector, changed as a function of the pulse number within a train, for two different runs with the MLLs realigned in between. The two horizontal dark lines seen in each image are the gaps between detector chips on the AGIPD. The runs consisted of 1000 trains, each with 30 pulses for a total of 30000 recorded frames. Figures 2 and 3 show ten images and twelve images of the pupil, respectively. The time sequence of images in both figures goes from left to right and top to bottom. The pupil is uniformly illuminated at the start in Figure 2 (start of one train), showing that the lenses were at the correct orientation to fulfill the Bragg condition for all points in the lens. However, the last few images in Figure 2 show a reduction of intensity in the bottom right-hand side of the pupil. The observed reduction is similar to patterns observed when the lenses are not properly aligned, and we speculated that this was caused by a small rotation of the lens, induced by the X-ray pulses in a train and returning to the original alignment by the start of the next train. To test this hypothesis, we changed the tilt of the lenses in such a way to observe an improvement of the diffraction efficiency during the course of the train. In Figure 3, at the start of the train the lenses seem to be out of the Bragg condition and become better aligned by the end of the train (last images). Due to the problems with gain switching on the detector the highest intensity parts are saturated $\left(5^{\text {th }}, 8^{\text {th }}, 9^{\text {th }}, 10^{\text {th }}, 11^{\text {th }}\right.$ and $12^{\text {th }}$ images $)$. Final run was done with 30 pulse trains and at $46.5 \%$ transmission. In this case the lens diffraction efficiency slowly drifted such that by the end of the 1000 trains no diffraction could be seen on the detector. Thus, we feared that both lenses were destroyed. However, this was not the case. We could verify that both lenses were still there with the $\mathrm{x}$-ray eye detector but no longer in the Bragg condition. After re-aligning them we saw that they were still diffracting.

After the experiment we examined both lenses with SEM and compared them with images taken before the experiment. One of these lenses before and after the experiment is displayed in Figure 4. No obvious changes could be seen in these SEM images. Later measurements of the diffraction of these lenses also did not show any change.
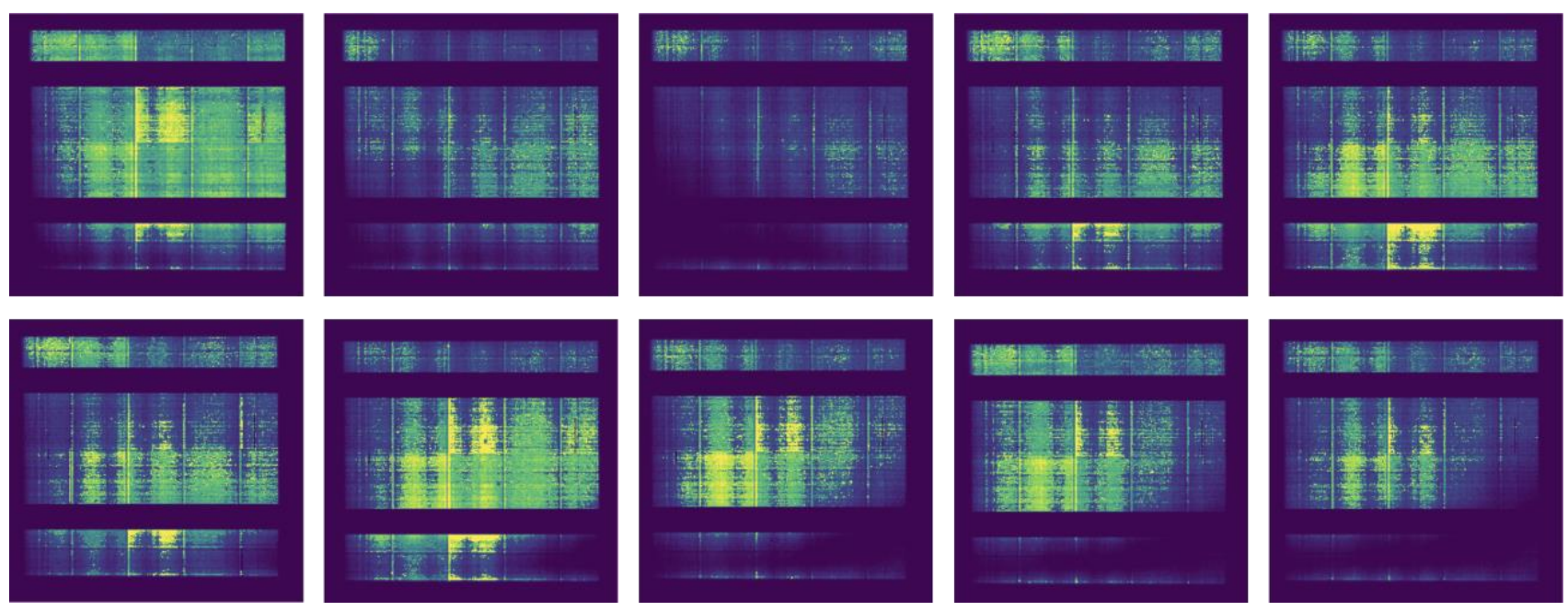

Figure 2. Images of the pupil (few snapshots) as a function of time along one train with 30 pulses and $23.5 \%$ transmission. The sequence of images with time goes from left to right and top to bottom. Ideal image should show uniformly illuminated square with two horizontal dark bars. Horizontal bars are gaps between the detector tiles.

In another test at even higher beamline transmission during experimental run 2543, we found that the upstream lens broke off its mount. One explanation for this and the wobbling of the lenses over the course of the pulse train is that the heat, caused by x-ray absorption of the lens, cannot be dissipated quickly enough. Although the lens appears to be thermally 
very stable the large heat gradients cause a tilt of the lens, and most probably a bending at the points at which they are attached to the diamond mount. There they were connected only via very small Pt welding dots (as indicated in Figure 4). This way of mounting the MLLs was used in synchrotron setups with no problems for many years. The conditions at XFELs are obviously much more demanding, and must be addressed to be able to prevent lens wobbling so that they can be used at high intensity for all pulses in a train. We demonstrated that the MLLs can focus, image and survive in European XFEL beam even at very high intensities. However, small welding spots connecting the MLLs to the diamond substrate proved to be the bottleneck for fast enough heat dissipation. We are currently pursuing new, more robust ways of mounting MLLs, which would keep them stable and under Bragg condition even at full beam intensity.
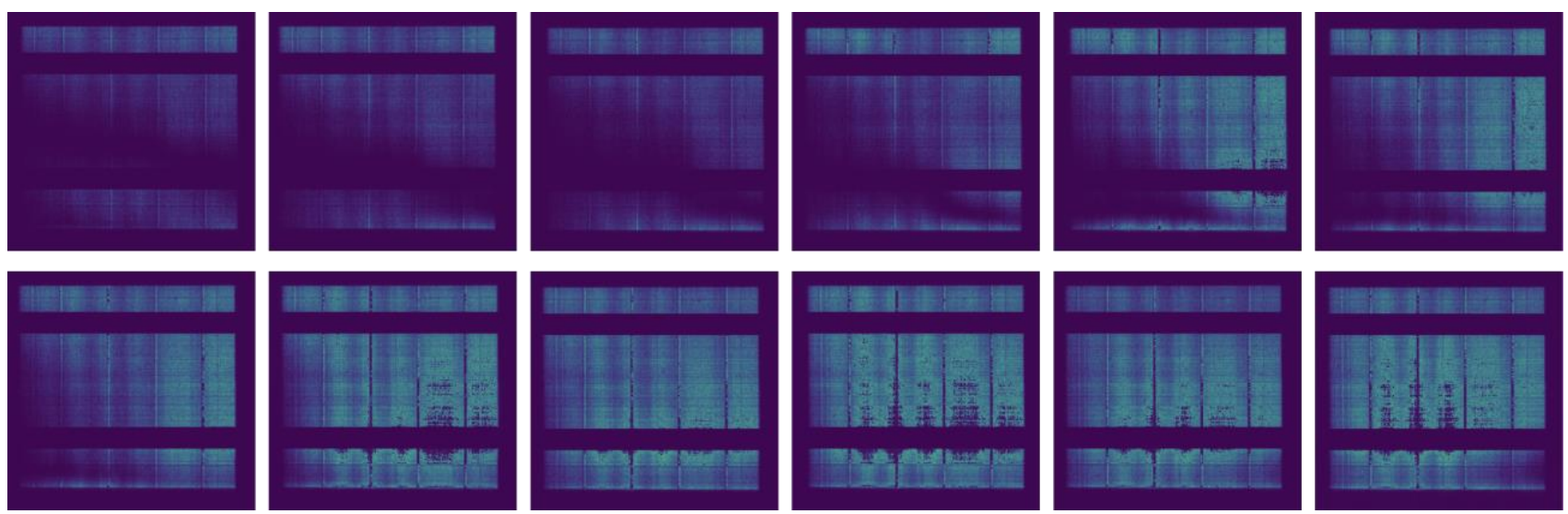

Figure 3. Images of the pupil (few snapshots) as a function of time along another (later) train with 30 pulses and $23.5 \%$ transmission. The sequence of images with time goes from left to right and top to bottom. Ideal image should show uniformly illuminated square with two horizontal dark bars. Horizontal bars are gaps between the detector tiles.
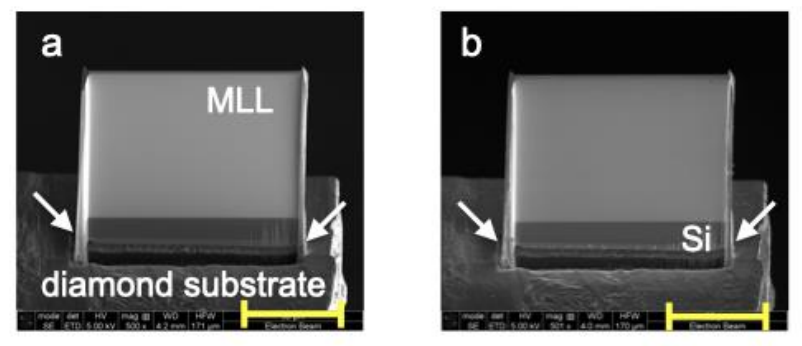

Figure 4. SEM image of one of the MLLs before (a) and after (b) XFEL experiment. An MLL attached to the Si substrate is affixed with platinum to the diamond substrate only in two locations as indicated by arrows. The size of the yellow scale bar is $50 \mu \mathrm{m}$.

\section{ACKNOWLEDGMENTS}

We would like to thank Sabrina Bolmer (DESY), Lars Gumprecht, Tjark Delmas, Julia Maracke (CFEL), Shiwani Sharma (The Hamburg Centre for Ultrafast Imaging), Huijong Han and Joachim Schulz (European XFEL) for technical assistance and help with experimental setup, Thomas Keller and Andreas Stierle (DESY) for access to FIB in Nanolab and Andrew Aquila (SLAC), Harald Sinn (European XFEL), Sang-Kil Son, Robin Santra (CFEL) and Ozgur Culfa (Univ. of NebraskaLincoln) for helpful discussions. This work was also supported by the Cluster of Excellence "CUI: Advanced Imaging of Matter" of the Deutsche Forschungsgemeinschaft (DFG) - EXC 2056 - project ID 390715994. 


\section{REFERENCES}

[1] Fuchs, M., Trigo, M., Chen, J., Ghimire, S., Scwartz, S., Kozina, M., Jian, M., Henighan, T., Bray C., Ndabashimiye, G., Bucksbaum, P. H., Feng, Y., Herrmann, S., Carini, G. A., Pines, J., Hart, P., Kenney, C., Gullet, S., Boutet, S., Williams, G. J., Messerschmidt, M. Seibert, M. M., Moeller, S., Hastings, J. B., and Reis, D. A., Nat. phys. 11, 964-970 (2015).

[2] Shwartz, S. Fuchs, M. Hastings, J. B., Inubushi, Y., Ishikawa, T., Katayama, T., Reis, D. A., Sato, T., Tono, K., Yabashi, M., Yudovich, S., and Harris, S. E., Phys. Rev. Lett. 112, 163901 (2014).

[3] Glover, T. E., Fritz, D. M., Cammarat, M., Allison, T. K., Coh S., Feldkamp, J. M., Lemke, H., Zhu, D., Feng, Y., Coffee, R. N., Fuchs, M., Chimire, S., Chen, J., Shwartz, S., Reis, D. A., Harris, S. E., and Hastings, J. B., Nature 488, 603-608 (2012).

[4] Tamasaku, K., Sewada, K., Nishibori, E., and Ishikawa, T., Nat. phys. 7, 705-708 (2011).

[5] Kang, H. C., Maser, J., Stephenson, G. B., Liu, C., Conley, R., Macrander, A. T., and Vogt, S., PRL 96, 127401 (2006)

[6] Prasciolu, M., and Bajt, S., Appl. Sci. 8, 571 (2018).

[7] Bajt, S., Prasciolu, M., Fleckenstein, H., Domaracký, M., Chapman, H. N., Morgan, A. J., Yefanov, O., Messerschmidt, M., Du, Y., Murray, K. T., Mariani, V., Kuhn, M., Aplin, S., Pande, K., Villanueva-Perez, P., Stachnik, K., Chen, J. P. J., Andrejczuk, A., Meents, A., Burkhardt, A., Pennicard, D., Huang, X., Yan, H., Nazaretski, E., Chu, Y. S., Hamm, C. E., Light: Science and Applications 7, e17162 (2018).

[8] Madsen, A., Hallmann, J., Roth, T., Ansaldi, G., "Technical Design Report: Scientific Instrument MID”, <https://bib-pubdb1.desy.de/record/154260> (2013).

[9] Madsen, A., Hallmann, J., Ansaldi, G., Roth, T., Lu, W., Kim, C., Boesenberg, U., Zozulya, A., Möller, J., Shayduk, R., Scholz, M., Bartmann, A., Schmidt, A., Lobato, I., Sukharnikov, K., Reiser, M., Kazariana, K., and Petrov,I., J. Synchroton. Rad. 28, 637 (2021).

[10] Tschentscher, T., Bressler, C., Grünert, J., Madsen, A., Mancuso, A., Meyer, M., Scherz, A., Sinn, H., and Zastrau, U., Appl. Sci. 7, 592 (2017).

[11] https://www.mdpi.com/2076-3417/7/6/592.

[12] Snigirev, A., Kohn, V., Snigireva, I., and Lengeler, B., Nature 384, 49-51 (1996).

[13] Bajt, S., and Schroer, C. G., [Sub-micrometer focusing and high-resolution imaging with refractive lenses and multilayer Laue optics], in Synchrotron Light Sources and Free-Electron Lasers, E. J. Jaeschke et al. (eds.), Springer, Cham, 1-25 (2019).

[14] Mimura, H., Yumoto, H., Matsuyama, S., Koyama, T., Tono, K., Inubushi, Y., Togashi, T., Sato, T., Kim, J., Fukui, R., Sano, Y., Yabashi, M., Ohashi, H., Ishikawa, T., Yamauchi, K., Nat. comm. 5, 3539 (2014).

[15] David, C., Gorelick, S., Rutishauser, S., Krzywinski, J., Vila-Comamala, J., Guzenko, V. A., Bunk, O., Färm, E., Ritala, M., Cammarata, M., Fritz, D. M., Barrett, R., Samoylova, L., Grünert, J., and Sinn, H., Sci. Rep. 1, 57 (2011).

[16] Nilsson, D., Uhlén, F., Reinspach, J., Hertz, H. M., Holmberg, A., Sinn, H., and Vogt, U., New J. Phys. 14, 043010 (2012).

[17] Rutishauser, S., Samoylova, L., Krzywinski, J., Bunk, O., Grünert, J., Sinn, H., Cammarata, M., Fritz, D.M., David, C., Nat. comm. 3, 947 (2012).

[18] Nilsson, D., Holmberg, A., Sinn, H., Vogt, U., Nucl. Instrum. Methods Phys. Res. A 621, 620-626 (2010).

[19] Makita, M., Karvinen, P., Guzenko, V. A., Kujala, N., Vagovic, P., David, C., Microelectr. Eng. 176, 75-78 (2017).

[20] Bajt, S. Prasciolu, M., Morgan, A. J., Chapman, H. N., Krzywinski, J., Andrejczuk, A., “One Dimensional Focusing with High Numerical Aperture Multilayer Laue Lens", XRM 2014: Proceedings of the 12th

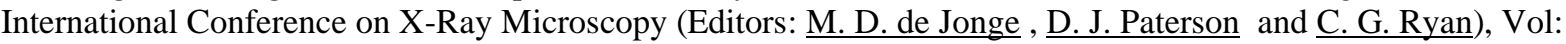
1696, 020049 (2016).

[21] Jensen, C. P., Madsen, K. K., Christensen, F. E., Exp. Astron. 20, 93-103 (2005).

[22] Fernández-Perea, M., Pivovaroff, M. J., Soufli, R., Alameda, J., Mirkarimi, P., Descalle, M. -A., Baker, S. L., McCarville, T., Ziock, K., Hornback, D., Romaine, S., Bruni, R., Zhong, Z., Honkimäki, V., Ziegler, E., Christensen, F. E., Jakobsen A. C., Nucl. Instrum. Methods Phys. Res. A 710, 114-119 (2013).

[23] Morgan, A. J., Prasciolu, M., Andrejczuk, A., Krzywinski, J., Meents, A., Pennicard, D., Graafsma, H., Barty, A., Bean, R. J., Barthelmess, M., Oberthuer, D., Yefanov, O., Aquila, A., Chapman, H. N., and Bajt, S., Sci. Rep. 5, 09892 (2015). 
[24] Prasciolu, M., Leontowich, A. F. G., Krzywinski, J., Andrejczuk, A., Chapman, H. N., and Bajt, S., Opt. Mater. Express 5, 748-755 (2015).

[25] <https://www.xfel.eu/facility/instruments/mid/index_eng.html>

[26] Yan, H. F., Huang, X. J., Bouet, N., Zhou, J., Nazaretski, E., and Chu, Y. S., Opt Express 25, 25234-25242 (2017).

[27] Allahgholi, A., Becker, J., Delfs, A., Dinapoli, R. Gttlicher, P., Graafsma, H., Greiffenberg, D., Hirsemann, H., Jack, S., Klyuev, A., Krger, H., Kuhn, M., Laurus, T., Marras, A., Mezza, D., Mozzanica, A., Poehlsen, J., Shalev, O. S., Sheviakov, I., Schmitt, B., Schwandt, J., Shi, X., Smoljanin, S., Trunk, U., Zhang, J., Zimmer, M., Nucl. Instrum. Methods Phys. Res. A 942, 162324 (2019).

[28] Morgan, A. J., Quiney, H. M., Bajt, S., and Chapman, H. N., J. Appl. Cryst. 53, 760-780 (2020).

[29] Morgan, A. J., Murray, K. T. , Prasciolu, M., Fleckenstein, H., Yefanov, O., Villanueva-Perez, P., Mariani, V., Domaracky, M., Kuhn, M., Aplin, S., Mohacsi, I., Messerschmidt, M., Stachnik, K., Du, Y., Burkhart, A., Meents, A., Nazaretski, E., Yan, H., Huang, X., Chu, Y., Chapman, H. N., and Bajt, S., J. Appl. Cryst. 53, 927-936 (2020). [30] Morgan, A. J., Murray, K. T., Quiney, H. M., Bajt, S., and Chapman, H. N., J. Appl. Cryst. 53, 1603-1612 (2020). 